\title{
Solutions for Integral Boundary Value Problems of Nonlinear Hadamard Fractional Differential Equations
}

\author{
Keyu Zhang $\mathbb{D}^{1}{ }^{1}$ Jianguo Wang $\mathbb{D}^{1},{ }^{1}$ and Wenjie $\mathrm{Ma}^{2}$ \\ ${ }^{1}$ School of Mathematics, Qilu Normal University, Jinan 250013, China \\ ${ }^{2}$ Department of Applied Mathematics, Shandong University of Science and Technology, Qingdao 266590, China \\ Correspondence should be addressed to Keyu Zhang; keyu_292@163.com
}

Received 25 August 2018; Accepted 19 October 2018; Published 1 November 2018

Academic Editor: Xinguang Zhang

Copyright (C) 2018 Keyu Zhang et al. This is an open access article distributed under the Creative Commons Attribution License, which permits unrestricted use, distribution, and reproduction in any medium, provided the original work is properly cited.

In this paper using fixed point methods we establish some existence theorems of positive (nontrivial) solutions for integral boundary value problems of nonlinear Hadamard fractional differential equations.

\section{Introduction}

In this work we study the following integral boundary value problems of nonlinear Hadamard fractional differential equations

$$
\begin{aligned}
D^{\beta}\left(\varphi_{p}\left(D^{\alpha} u(t)\right)\right) & =f(t, u(t)), \quad 1<t<e, \\
u(1) & =u^{\prime}(1)=u^{\prime}(e)=0, \\
D^{\alpha} u(1) & =0, \\
\varphi_{p}\left(D^{\alpha} u(e)\right) & =\mu \int_{1}^{e} \varphi_{p}\left(D^{\alpha} u(t)\right) \frac{\mathrm{d} t}{t},
\end{aligned}
$$

where $\alpha, \beta$, and $\mu$ are three positive real numbers with $\alpha \in$ $(2,3], \beta \in(1,2]$, and $\mu \in[0, \beta), \varphi_{p}(s)=|s|^{p-2} s$ is the $p$ Laplacian for $p>1, s \in \mathbb{R}$, and $f$ is a continuous function on $[1, e] \times \mathbb{R}$. Moreover, let $\varphi_{p}^{-1}=\varphi_{q}$ with $1 / p+1 / q=1$. In what follows, we offer some related definitions and lemmas for Hadamard fractional calculus.

Definition 1 (see [1, Page 111]). The $\alpha$ th Hadamard fractional order derivative of a function $y:[1,+\infty) \longrightarrow \mathbb{R}$ is defined by

$$
D^{\alpha} y(t)=\frac{1}{\Gamma(n-\alpha)}\left(t \frac{\mathrm{d}}{\mathrm{d} t}\right)^{n} \int_{1}^{t}\left(\log \frac{t}{s}\right)^{n-\alpha-1} y(s) \frac{\mathrm{d} s}{s}
$$

where $\alpha>0, n=[\alpha]+1$, and $[\alpha]$ denotes the largest integer which is less than or equal to $\alpha$. Moreover, we here also offer the $\alpha$ th Hadamard fractional order integral of $y$ : $[1,+\infty) \longrightarrow \mathbb{R}$ which is defined by

$$
I^{\alpha} y(t)=\frac{1}{\Gamma(\alpha)} \int_{1}^{t}\left(\log \frac{t}{s}\right)^{\alpha-1} y(s) \frac{\mathrm{d} s}{s}
$$

where $\Gamma$ is the gamma function.

Lemma 2 (see $[1$, Theorem 2.3]). Let $\alpha>0, n=[\alpha]+1$. Then

$$
\begin{aligned}
I^{\alpha} D^{\alpha} y(t)= & y(t)+c_{1}(\log t)^{\alpha-1}+c_{2}(\log t)^{\alpha-2}+\cdots \\
& +c_{n}(\log t)^{\alpha-n}
\end{aligned}
$$

where $c_{i} \in \mathbb{R}, i=1,2, \ldots, n$.

In recent years, there have been some significant developments in the study of boundary value problems for nonlinear fractional differential equations; we refer to [2-11] and the references therein. For more related works, see also [12-49]. For example, by using monotone iterative methods, Wang et al. [3] investigated a class of boundary value problems of Hadamard fractional differential equations involving nonlocal multipoint discrete and Hadamard integral boundary conditions and established monotone iterative sequences, which can converge to the unique positive solution of their problems. Similar methods are also applied in [4, 5, 12-15].

For differential equations with the $p$-Laplacian, see, for example, $[6,7,15-20]$ and the references therein. In [6], Wang 
considered the nonlinear Hadamard fractional differential equation with integral boundary condition and $p$-Laplacian operator

$$
\begin{aligned}
D^{\beta} \varphi_{p}\left(D^{\alpha} u(t)\right) & =f(t, u(t)), \quad t \in(1, T), \\
u(T) & =\lambda I^{\sigma} u(\eta), \\
D^{\alpha} u(1) & =0 \\
u(1) & =0
\end{aligned}
$$

where $f$ grows $(p-1)$-sublinearly at $+\infty$, and by using the Schauder fixed point theorem, a solution existence result is obtained. In [7], $\mathrm{Li}$ and Lin used the Guo-Krasnosel'skii fixed point theorem to obtain the existence and uniqueness of positive solutions for (1) with $\mu=0$.

However, we note that these are seldom considered Hadamard fractional differential equations with the $p$ Laplacian in the literature; in this paper we are devoted to this direction. We first utilize the Guo-Krasnosel'skii fixed point theorem to obtain two positive solutions existence theorems when $f$ grows $(p-1)$-superlinearly and $(p-1)$-sublinearly with the $p$-Laplacian, and secondly by using the fixed point index, we obtain a nontrivial solution existence theorem without the $p$-Laplacian, but the nonlinearity can allow being sign-changing and unbounded from below. This improves and generalizes some semipositone problems [21-31].

\section{Preliminaries}

In this section, we first calculate Green's functions associated with (1) and then transform the boundary value problem into its integral form. For this, we give the following lemma.

Lemma 3. Let $\alpha, \beta, \mu, \varphi_{p}$, and $D^{\alpha}, D^{\beta}$ be as in (1). Then (1) can take the integral form

$$
u(t)=\int_{1}^{e} G(t, s) \varphi_{q}\left(\int_{1}^{e} H(s, \tau) f(\tau, u(\tau)) \frac{\mathrm{d} \tau}{\tau}\right) \frac{\mathrm{d} s}{s}
$$

$$
\text { for } t \in[1, e] \text {, }
$$

where

$$
\begin{aligned}
& G(t, s)=\frac{1}{\Gamma(\alpha)} \\
& \quad \begin{cases}(\log t)^{\alpha-1}(1-\log s)^{\alpha-2}-(\log t-\log s)^{\alpha-1}, & 1 \leq s \leq t \leq e, \\
(\log t)^{\alpha-1}(1-\log s)^{\alpha-2}, & 1 \leq t \leq s \leq e,\end{cases}
\end{aligned}
$$

and

$$
\begin{aligned}
& H(t, \tau)=H_{1}(t, \tau)+\frac{\mu}{(\beta-\mu) \Gamma(\beta)}(\log t)^{\beta-1} \log \tau(1-\log \tau)^{\beta-1}, \quad \text { for } t, \tau \in[1, e], \\
& H_{1}(t, \tau)=\frac{1}{\Gamma(\beta)} \begin{cases}(\log t)^{\beta-1}(1-\log \tau)^{\beta-1}-(\log t-\log \tau)^{\beta-1}, & 1 \leq \tau \leq t \leq e \\
(\log t)^{\beta-1}(1-\log \tau)^{\beta-1}, & 1 \leq t \leq \tau \leq e\end{cases}
\end{aligned}
$$

Proof. Use $y(t)$ to replace $f(t, u)$ in (1). Let $D^{\beta}\left(\varphi_{p}\left(D^{\alpha} u(t)\right)\right)=$ $y(t)$. Then from Lemma 2 we have

$$
\begin{array}{r}
\varphi_{p}\left(D^{\alpha} u(t)\right)=I^{\beta} y(t)+c_{1}(\log t)^{\beta-1}+c_{2}(\log t)^{\beta-2}, \\
\text { for } c_{i} \in \mathbb{R}, i=1,2 .
\end{array}
$$

Note that $D^{\alpha} u(1)=0$ implies $\varphi_{p}\left(D^{\alpha} u(1)\right)=0$, and then $c_{2}=$ 0 . Therefore, we obtain

$$
\varphi_{p}\left(D^{\alpha} u(t)\right)=I^{\beta} y(t)+c_{1}(\log t)^{\beta-1} .
$$

Next, we calculate $\varphi_{p}\left(D^{\alpha} u(e)\right)$ and $\mu \int_{1}^{e} \varphi_{p}\left(D^{\alpha} u(t)\right)(\mathrm{d} t / t)$ :

$$
\begin{aligned}
\varphi_{p}\left(D^{\alpha} u(e)\right) & =I^{\beta} y(e)+c_{1} \\
& =c_{1}+\frac{1}{\Gamma(\beta)} \int_{1}^{e}(1-\log \tau)^{\beta-1} y(\tau) \frac{\mathrm{d} \tau}{\tau}
\end{aligned}
$$

and

$$
\begin{aligned}
& \mu \int_{1}^{e} \varphi_{p}\left(D^{\alpha} u(t)\right) \frac{\mathrm{d} t}{t} \\
& \quad=\mu \int_{1}^{e} I^{\beta} y(t) \frac{\mathrm{d} t}{t}+\mu c_{1} \int_{1}^{e}(\log t)^{\beta-1} \frac{\mathrm{d} t}{t}
\end{aligned}
$$

$$
=\frac{\mu c_{1}}{\beta}+\frac{\mu}{\Gamma(\beta)} \int_{1}^{e} \int_{1}^{t}(\log t-\log \tau)^{\beta-1} y(\tau) \frac{\mathrm{d} \tau}{\tau} \frac{\mathrm{d} t}{t} .
$$

The condition $\varphi_{p}\left(D^{\alpha} u(e)\right)=\mu \int_{1}^{e} \varphi_{p}\left(D^{\alpha} u(t)\right)(\mathrm{d} t / t)$ enables us to obtain

$$
\begin{aligned}
c_{1}= & \frac{\mu \beta}{(\beta-\mu) \Gamma(\beta)} \int_{1}^{e} \int_{1}^{t}(\log t-\log \tau)^{\beta-1} y(\tau) \frac{\mathrm{d} \tau}{\tau} \frac{\mathrm{d} t}{t} \\
& -\frac{\beta}{(\beta-\mu) \Gamma(\beta)} \int_{1}^{e}(1-\log \tau)^{\beta-1} y(\tau) \frac{\mathrm{d} \tau}{\tau} .
\end{aligned}
$$

Substituting $c_{1}$ into (10) gives

$$
\begin{aligned}
\varphi_{p} & \left(D^{\alpha} u(t)\right)=\frac{1}{\Gamma(\beta)} \int_{1}^{t}(\log t-\log \tau)^{\beta-1} y(\tau) \frac{\mathrm{d} \tau}{\tau} \\
& -\frac{\beta(\log t)^{\beta-1}}{(\beta-\mu) \Gamma(\beta)} \int_{1}^{e}(1-\log \tau)^{\beta-1} y(\tau) \frac{\mathrm{d} \tau}{\tau} \\
+ & \frac{\mu \beta(\log t)^{\beta-1}}{(\beta-\mu) \Gamma(\beta)} \int_{1}^{e} \int_{1}^{t}(\log t-\log \tau)^{\beta-1} y(\tau) \frac{\mathrm{d} \tau}{\tau} \frac{\mathrm{d} t}{t}
\end{aligned}
$$




$$
\begin{aligned}
& =\frac{1}{\Gamma(\beta)} \int_{1}^{t}(\log t-\log \tau)^{\beta-1} y(\tau) \frac{\mathrm{d} \tau}{\tau}-\frac{(\log t)^{\beta-1}}{\Gamma(\beta)} \\
& \cdot \int_{1}^{e}(1-\log \tau)^{\beta-1} y(\tau) \frac{\mathrm{d} \tau}{\tau}+\frac{(\log t)^{\beta-1}}{\Gamma(\beta)} \\
& \cdot \int_{1}^{e}(1-\log \tau)^{\beta-1} y(\tau) \frac{\mathrm{d} \tau}{\tau}-\frac{\beta(\log t)^{\beta-1}}{(\beta-\mu) \Gamma(\beta)} \\
& \cdot \int_{1}^{e}(1-\log \tau)^{\beta-1} y(\tau) \frac{\mathrm{d} \tau}{\tau}+\frac{\mu \beta(\log t)^{\beta-1}}{(\beta-\mu) \Gamma(\beta)} \\
& \cdot \int_{1}^{e} \int_{1}^{t}(\log t-\log \tau)^{\beta-1} y(\tau) \frac{\mathrm{d} \tau}{\tau} \frac{\mathrm{d} t}{t}=-\frac{1}{\Gamma(\beta)} \\
& \cdot \int_{1}^{t}\left[(\log t)^{\beta-1}(1-\log \tau)^{\beta-1}-(\log t-\log \tau)^{\beta-1}\right] \\
& \cdot y(\tau) \frac{\mathrm{d} \tau}{\tau}-\frac{1}{\Gamma(\beta)} \int_{t}^{e}(\log t)^{\beta-1}(1-\log \tau)^{\beta-1} \\
& y(\tau) \frac{\mathrm{d} \tau}{\tau}+\frac{\mu \beta(\log t)^{\beta-1}}{(\beta-\mu) \Gamma(\beta)} \\
& \cdot \int_{1}^{e} \int_{1}^{t}(\log t-\log \tau)^{\beta-1} y(\tau) \frac{\mathrm{d} \tau}{\tau} \frac{\mathrm{d} t}{t} \\
& -\frac{\mu(\log t)^{\beta-1}}{(\beta-\mu) \Gamma(\beta)} \int_{1}^{e}(1-\log \tau)^{\beta-1} y(\tau) \frac{\mathrm{d} \tau}{\tau} \\
& =-\int_{1}^{e} H_{1}(t, \tau) y(\tau) \frac{\mathrm{d} \tau}{\tau}+\frac{\mu \beta(\log t)^{\beta-1}}{(\beta-\mu) \Gamma(\beta)} \\
& \cdot \int_{1}^{e} \int_{\tau}^{e}(\log t-\log \tau)^{\beta-1} y(\tau) \frac{\mathrm{d} t}{t} \frac{\mathrm{d} \tau}{\tau} \\
& -\frac{\mu(\log t)^{\beta-1}}{(\beta-\mu) \Gamma(\beta)} \int_{1}^{e}(1-\log \tau)^{\beta-1} y(\tau) \frac{\mathrm{d} \tau}{\tau} \\
& =-\int_{1}^{e} H_{1}(t, \tau) y(\tau) \frac{\mathrm{d} \tau}{\tau}+\frac{\mu(\log t)^{\beta-1}}{(\beta-\mu) \Gamma(\beta)} \\
& \cdot \int_{1}^{e}(1-\log \tau)^{\beta} y(\tau) \frac{\mathrm{d} \tau}{\tau}-\frac{\mu(\log t)^{\beta-1}}{(\beta-\mu) \Gamma(\beta)} \\
& \cdot \int_{1}^{e}(1-\log \tau)^{\beta-1} y(\tau) \frac{\mathrm{d} \tau}{\tau}=-\int_{1}^{e} H_{1}(t, \tau) \\
& y(\tau) \frac{\mathrm{d} \tau}{\tau}-\int_{1}^{e} \frac{\mu}{(\beta-\mu) \Gamma(\beta)}(\log t)^{\beta-1} \\
& \cdot \log \tau(1-\log \tau)^{\beta-1} y(\tau) \frac{\mathrm{d} \tau}{\tau}=-\int_{1}^{e} H(t, \tau) \\
& y(\tau) \frac{\mathrm{d} \tau}{\tau}
\end{aligned}
$$

Note that $-\varphi_{p}\left(D^{\alpha} u(t)\right)=\varphi_{p}\left(-D^{\alpha} u(t)\right)$, and hence we obtain

$$
-D^{\alpha} u(t)=\varphi_{q}\left(\int_{1}^{e} H(t, \tau) y(\tau) \frac{\mathrm{d} \tau}{\tau}\right),
$$

for $\alpha \in(2,3], t \in[1, e]$.

Then, if we let $x(t)=\varphi_{q}\left(\int_{1}^{e} H(t, \tau) y(\tau)(\mathrm{d} \tau / \tau)\right), t \in[1, e]$, from Lemma 2 we obtain

$$
\begin{aligned}
u(t)= & -I^{\alpha} x(t)+c_{1}(\log t)^{\alpha-1}+c_{2}(\log t)^{\alpha-2} \\
& +c_{3}(\log t)^{\alpha-3}, \quad \text { for } c_{i} \in \mathbb{R}, i=1,2,3 .
\end{aligned}
$$

The condition $u(1)=u^{\prime}(1)=0$ implies that $c_{2}=c_{3}=0$. Then we substitute $e$ into the first derivative of $u$, and we calculate $c_{1}$ as follows:

$$
c_{1}=\frac{1}{\Gamma(\alpha)} \int_{1}^{e}(1-\log s)^{\alpha-2} x(s) \frac{\mathrm{d} s}{s} .
$$

As a result, from (16) we have

$$
\begin{aligned}
u(t)= & -\frac{1}{\Gamma(\alpha)} \int_{1}^{t}(\log t-\log s)^{\alpha-1} x(s) \frac{\mathrm{d} s}{s} \\
& +\frac{1}{\Gamma(\alpha)} \int_{1}^{e}(\log t)^{\alpha-1}(1-\log s)^{\alpha-2} x(s) \frac{\mathrm{d} s}{s} \\
= & \int_{1}^{e} G(t, s) \varphi_{q}\left(\int_{1}^{e} H(s, \tau) y(\tau) \frac{\mathrm{d} \tau}{\tau}\right) \frac{\mathrm{d} s}{s},
\end{aligned}
$$

for $t \in[1, e]$.

This completes the proof.

Lemma 4. Green's functions $G, H$ defined by (7) and (8) have the following properties:

(i) $G, H$ are continuous, nonnegative functions on $[1, e] \times$ $[1, e]$,

(ii) $(\log t)^{\alpha-1}\left[(1-\log s)^{\alpha-2}-(1-\log s)^{\alpha-1}\right] \leq \Gamma(\alpha) G(t, s) \leq$ $(1-\log s)^{\alpha-2}-(1-\log s)^{\alpha-1}$, for $t, s \in[1, e]$.

From [7, Lemma 7] and [8, Lemma 2.2] we easily obtain this lemma, so we omit its proof.

$$
\begin{aligned}
& \text { Let } \\
& \begin{array}{l}
G_{1}(t, s)=\int_{1}^{e} G(t, \tau) H(\tau, s) \frac{\mathrm{d} \tau}{\tau}, \\
\phi(s)=\frac{1}{\Gamma(\alpha)} \\
\cdot \int_{1}^{e}\left[(1-\log t)^{\alpha-2}-(1-\log t)^{\alpha-1}\right] H(t, s) \frac{\mathrm{d} t}{t}, \\
\text { for } t, s \in[1, e] .
\end{array}
\end{aligned}
$$

Then we obtain the following lemma.

Lemma 5. There exist $\kappa_{1}=\int_{1}^{e}(\log t)^{\alpha-1} \phi(t)(\mathrm{d} t / t), \kappa_{2}=$ $\int_{1}^{e} \phi(t)(\mathrm{d} t / t)$ such that

$$
\kappa_{1} \phi(s) \leq \int_{1}^{e} G_{1}(t, s) \phi(t) \frac{\mathrm{d} t}{t} \leq \kappa_{2} \phi(s),
$$


Proof. We only prove the left inequality above. From Lemma 4(ii) we have

$$
\begin{aligned}
& \int_{1}^{e} G_{1}(t, s) \phi(t) \frac{\mathrm{dt}}{t}=\int_{1}^{e} \int_{1}^{e} G(t, \tau) \\
& \cdot H(\tau, s) \frac{\mathrm{d} \tau}{\tau} \phi(t) \frac{\mathrm{d} t}{t} \geq \frac{1}{\Gamma(\alpha)} \\
& \cdot \int_{1}^{e} \int_{1}^{e}(\log t)^{\alpha-1}\left[(1-\log \tau)^{\alpha-2}-(1-\log \tau)^{\alpha-1}\right] \\
& \cdot H(\tau, s) \frac{\mathrm{d} \tau}{\tau} \phi(t) \frac{\mathrm{d} t}{t}=\kappa_{1} \phi(s) .
\end{aligned}
$$

This completes the proof.

Let $\mathscr{E}=C[1, e]$ be the Banach space equipped with the norm $\|u\|=\max _{t \in[1, e]}|u(t)|$. Then we define two sets on $\mathscr{E}$ as follows:

$$
\begin{aligned}
P & =\{u \in \mathscr{E}: u(t) \geq 0, \forall t \in[1, e]\}, \\
P_{0} & =\left\{u \in \mathscr{E}: u(t) \geq(\log t)^{\alpha-1}\|u\|, \forall t \in[1, e]\right\} .
\end{aligned}
$$

Consequently, $P, P_{0}$ are cones on $\mathscr{E}$. From Lemma 3 we can define an operator $A$ on $\mathscr{E}$ as follows:

$(A u)(t)$

$$
=\int_{1}^{e} G(t, s) \varphi_{q}\left(\int_{1}^{e} H(s, \tau) f(\tau, u(\tau)) \frac{\mathrm{d} \tau}{\tau}\right) \frac{\mathrm{d} s}{s},
$$

for $u \in \mathscr{E}, t \in[1, e]$.

The continuity of $G, H, f$ implies that $A: \mathscr{E} \longrightarrow \mathscr{E}$ is a completely continuous operator and the existence of solutions for (1) if and only if the existence of fixed points for $A$.

Lemma 6 (see [50]). Let $\mathscr{E}$ be a Banach space and $\Omega$ a bounded open set in $\mathscr{E}$. Suppose that $A: \Omega \longrightarrow \mathscr{E}$ is a continuous compact operator. If there exists $u_{0} \in E \backslash\{0\}$ such that

$$
u-A u \neq \mu u_{0}, \quad \forall u \in \partial \Omega, \quad \mu \geq 0,
$$

then the topological degree $\operatorname{deg}(I-A, \Omega, 0)=0$.

Lemma 7 (see [50]). Let $\mathscr{E}$ be a Banach space and $\Omega$ a bounded open set in $\mathscr{E}$ with $0 \in \Omega$. Suppose that $A: \Omega \longrightarrow \mathscr{E}$ is a continuous compact operator. If

$$
A u \neq \mu u, \quad \forall u \in \partial \Omega, \mu \geq 1,
$$

then the topological degree $\operatorname{deg}(I-A, \Omega, 0)=1$.

Lemma 8 (see [50]). Let $\mathscr{E}$ be a Banach space and $P \subset \mathscr{E}$ a cone in $\mathscr{E}$. Assume that $\Omega_{1}, \Omega_{2}$ are open subsets of $\mathscr{E}$ with $0 \in \Omega_{1} \subset \bar{\Omega}_{1} \subset \Omega_{2}$, and let $A: P \cap\left(\bar{\Omega}_{2} \backslash \Omega_{1}\right) \rightarrow P$ be a completely continuous operator such that either

(G1) $\|A u\| \leq\|u\|, u \in \partial \Omega_{1} \cap P$, and $\|A u\| \geq\|u\|, u \in$ $\partial \Omega_{2} \cap P$,

or

(G2) $\|A u\| \geq\|u\|, u \in \partial \Omega_{1} \cap P$, and $\|A u\| \leq\|u\|, u \in$ $\partial \Omega_{2} \cap P$.

Then $A$ has a fixed point in $P \cap\left(\bar{\Omega}_{2} \backslash \Omega_{1}\right)$.

\section{Positive Solutions for (1)}

Let $B_{\varrho}:=\{u \in \mathscr{E}:\|u\|<\varrho\}$ for $\varrho>0$. Now, we first list our assumptions on $f$ :

(H1) $f \in C\left([0,1] \times \mathbb{R}^{+}, \mathbb{R}^{+}\right)$,

(H2) there exist $\delta_{1} \in(1, e), t_{0} \in(1, e)$ such that $\liminf _{u \longrightarrow+\infty}\left(f(t, u) / \varphi_{p}(u)\right) \geq \varphi_{p}\left(N_{1}\right), \liminf _{u \longrightarrow 0^{+}}(f(t, u) /$ $\left.\varphi_{p}(u)\right) \geq \varphi_{p}\left(N_{2}\right)$, uniformly on $t \in\left[\delta_{1}, e\right]$, where $2 N_{1}^{-1}$, $N_{2}^{-1} \in\left(0,\left(\log \delta_{1}\right)^{\alpha-1} \int_{1}^{e} G\left(t_{0}, s\right) \varphi_{q}\left(\int_{\delta_{1}}^{e} H(s, \tau)(\mathrm{d} \tau / \tau)\right)(\mathrm{d} s / s)\right)$,

(H3) there exists $\rho_{1}>0$ such that $f(t, u) \leq \varphi_{p}\left(N_{3} \rho_{1}\right)$, $\forall u \in\left[0, \rho_{1}\right], t \in[1, e]$, where $N_{3}^{-1}>\int_{1}^{e} G(e, s) \varphi_{q}\left(\int_{1}^{e} H(s\right.$, $\tau)(\mathrm{d} \tau / \tau))(\mathrm{d} s / s)$,

(H4) $\lim \sup _{u \rightarrow+\infty}\left(f(t, u) / \varphi_{p}(u)\right) \leq \varphi_{p}\left(M_{1}\right)$, $\limsup \operatorname{su}_{u \rightarrow 0^{+}}\left(f(t, u) / \varphi_{p}(u)\right) \leq \varphi_{p}\left(M_{2}\right)$, uniformly on $t \in[1, e]$, where $\left(2 M_{1}\right)^{-1}, M_{2}^{-1}>\int_{1}^{e} G(e, s) \varphi_{q}\left(\int_{1}^{e} H(s, \tau)(\mathrm{d} \tau /\right.$ $\tau))(\mathrm{d} s / s)$

(H5) there exist $\rho_{2}>0, \delta_{1} \in(1, e), t_{0} \in(1, e)$ such that $f(t, u) \geq \varphi_{p}\left(M_{3} \rho_{2}\right), \forall u \in\left[\left(\log \delta_{1}\right)^{\alpha-1} \rho_{2}, \rho_{2}\right], t \in\left[\delta_{1}, e\right]$, where

$$
M_{3}^{-1} \in\left(0, \int_{1}^{e} G\left(t_{0}, s\right) \varphi_{q}\left(\int_{\delta_{1}}^{e} H(s, \tau) \frac{\mathrm{d} \tau}{\tau}\right) \frac{\mathrm{d} s}{s}\right) .
$$

Lemma 9. Suppose that (H1) holds. Then $A(P) \subset P_{0}$.

Proof. If $u \in P$, from Lemma 4 we have

$$
\begin{gathered}
(A u)(t) \leq \frac{1}{\Gamma(\alpha)} \int_{1}^{e}\left[(1-\log s)^{\alpha-2}-(1-\log s)^{\alpha-1}\right] \\
\cdot \varphi_{q}\left(\int_{1}^{e} H(s, \tau) f(\tau, u(\tau)) \frac{\mathrm{d} \tau}{\tau}\right) \frac{\mathrm{d} s}{s}
\end{gathered}
$$

$\forall t \in[1, e]$.

On the other hand,

$$
\begin{aligned}
& (A u)(t) \geq(\log t)^{\alpha-1} \cdot \frac{1}{\Gamma(\alpha)} \\
& \cdot \int_{1}^{e}\left[(1-\log s)^{\alpha-2}-(1-\log s)^{\alpha-1}\right] \\
& \cdot \varphi_{q}\left(\int_{1}^{e} H(s, \tau) f(\tau, u(\tau)) \frac{\mathrm{d} \tau}{\tau}\right) \frac{\mathrm{d} s}{s} \\
& \geq(\log t)^{\alpha-1}\|A u\|, \quad \forall t \in[1, e] .
\end{aligned}
$$

This completes the proof.

Remark 10. Our aim is to find operator equation $u=A u$ has fixed points in $P$, and from Lemma 9, these fixed points must belong to the cone $P_{0}$. Therefore, our work space can be chosen $P_{0}$ rather than $P$.

In what follows, we discuss the existence of positive solutions for (1) in $P_{0}$.

Theorem 11. Suppose that (H1)-(H3) hold. Then (1) has at least two positive solutions. 
Proof. From (H3), when $u \in \partial B_{\rho_{1}} \cap P_{0}$, we have

$(A u)(t)$

$$
\begin{aligned}
& \leq \max _{t \in[1, e]} \int_{1}^{e} G(t, s) \varphi_{q}\left(\int_{1}^{e} H(s, \tau) f(\tau, u(\tau)) \frac{\mathrm{d} \tau}{\tau}\right) \frac{\mathrm{d} s}{s} \\
& \leq \int_{1}^{e} G(e, s) \varphi_{q}\left(\int_{1}^{e} H(s, \tau) \varphi_{p}\left(N_{3} \rho_{1}\right) \frac{\mathrm{d} \tau}{\tau}\right) \frac{\mathrm{d} s}{s} \\
& =N_{3} \rho_{1} \int_{1}^{e} G(e, s) \varphi_{q}\left(\int_{1}^{e} H(s, \tau) \frac{\mathrm{d} \tau}{\tau}\right) \frac{\mathrm{d} s}{s}<\rho_{1},
\end{aligned}
$$

$$
\forall t \in[1, e] .
$$

Hence, we obtain

$$
\|A u\|<\|u\|, \quad \text { for } u \in \partial B_{\rho_{1}} \cap P_{0} .
$$

On the other hand, by the second limit inequality in $(\mathrm{H} 2)$, there exists $r_{1} \in\left(0, \rho_{1}\right)$ such that

$$
f(t, u) \geq \varphi_{p}\left(N_{2} u\right), \quad \forall u \in\left[0, r_{1}\right], t \in\left[\delta_{1}, e\right] .
$$

Note that if $u \in \partial B_{r_{1}} \cap P_{0}, t \in\left[\delta_{1}, e\right]$, from the definition of $P_{0}$ we have

$$
u(t) \geq\left(\log \delta_{1}\right)^{\alpha-1}\|u\| .
$$

This, together with (31), implies that

$$
\begin{aligned}
& \|A u\|=\max _{t \in[1, e]}(A u)(t) \geq(A u)\left(t_{0}\right)=\int_{1}^{e} G\left(t_{0}, s\right) \\
& \cdot \varphi_{q}\left(\int_{1}^{e} H(s, \tau) f(\tau, u(\tau)) \frac{\mathrm{d} \tau}{\tau}\right) \frac{\mathrm{d} s}{s} \\
& \geq \int_{1}^{e} G\left(t_{0}, s\right) \varphi_{q}\left(\int_{\delta_{1}}^{e} H(s, \tau)\right. \\
& \left.\cdot \varphi_{p}\left(N_{2}\left(\log \delta_{1}\right)^{\alpha-1}\|u\|\right) \frac{\mathrm{d} \tau}{\tau}\right) \frac{\mathrm{d} s}{s} \\
& =N_{2}\left(\log \delta_{1}\right)^{\alpha-1}\|u\| \int_{1}^{e} G\left(t_{0}, s\right) \\
& \cdot \varphi_{q}\left(\int_{\delta_{1}}^{e} H(s, \tau) \frac{\mathrm{d} \tau}{\tau}\right) \frac{\mathrm{d} s}{s}>\|u\|,
\end{aligned}
$$

$$
\text { for } u \in \partial B_{r_{1}} \cap P_{0} \text {. }
$$

By the first limit inequality in (H2), there exist $R_{1}>\rho_{1}$ and $C_{1}>0$ such that

$$
f(t, u) \geq \varphi_{p}\left(N_{1} u\right)-C_{1}, \quad \forall u \in \mathbb{R}^{+}, t \in\left[\delta_{1}, e\right] .
$$

Note that $R_{1}$ can be chosen large enough, and if $u \in \partial B_{R_{1}} \cap P_{0}$, together with (32), there exists $C_{2}>0$ such that

$$
f(t, u) \geq \varphi_{p}\left(N_{1}\left(\log \delta_{1}\right)^{\alpha-1} R_{1}-C_{2}\right),
$$

Combining this and (33), we find

$$
\begin{gathered}
\|A u\| \geq \int_{1}^{e} G\left(t_{0}, s\right) \varphi_{q}\left(\int_{\delta_{1}}^{e} H(s, \tau)\right. \\
\left.\cdot \varphi_{p}\left(N_{1}\left(\log \delta_{1}\right)^{\alpha-1} R_{1}-C_{2}\right) \frac{\mathrm{d} \tau}{\tau}\right) \frac{\mathrm{d} s}{s} \\
=\left(N_{1}\left(\log \delta_{1}\right)^{\alpha-1} R_{1}-C_{2}\right) \int_{1}^{e} G\left(t_{0}, s\right) \\
\cdot \varphi_{q}\left(\int_{\delta_{1}}^{e} H(s, \tau) \frac{\mathrm{d} \tau}{\tau}\right) \frac{\mathrm{d} s}{s} \geq 2 R_{1}-C_{3},
\end{gathered}
$$

where $C_{3}=C_{2} \int_{1}^{e} G\left(t_{0}, s\right) \varphi_{q}\left(\int_{\delta_{1}}^{e} H(s, \tau)(\mathrm{d} \tau / \tau)\right)(\mathrm{d} s / s)$. Consequently, we have

$$
\|A u\|>\|u\|, \quad \text { for } \partial B_{R_{1}} \cap P_{0}, \text { if }\|u\| \longrightarrow \infty .
$$

In summary, from (30), (33), and (37) with $R_{1}>\rho_{1}>r_{1}$, Lemma 8 enables us to obtain that (1) has at least two positive solutions in $\left(\bar{B}_{R_{1}} \backslash B_{\rho_{1}}\right) \cap P_{0}$ and $\left(\bar{B}_{\rho_{1}} \backslash B_{r_{1}}\right) \cap P_{0}$. This completes the proof.

Theorem 12. Suppose that (H1), (H4)-(H5) hold. Then (1) has at least two positive solutions.

Proof. If $u \in \partial B_{\rho_{2}} \cap P_{0}$, we have $\|u\|=\rho_{2}$, and $u \in$ $\left[\left(\log \delta_{1}\right)^{\alpha-1} \rho_{2}, \rho_{2}\right]$, for $u \in P_{0}, t \in\left[\delta_{1}, e\right]$. Hence, from (H5) we obtain

$\|A u\|$

$$
\begin{aligned}
& \geq \int_{1}^{e} G\left(t_{0}, s\right) \varphi_{q}\left(\int_{1}^{e} H(s, \tau) f(\tau, u(\tau)) \frac{\mathrm{d} \tau}{\tau}\right) \frac{\mathrm{d} s}{s} \\
& \geq \int_{1}^{e} G\left(t_{0}, s\right) \varphi_{q}\left(\int_{\delta_{1}}^{e} H(s, \tau) \varphi_{p}\left(M_{3} \rho_{2}\right) \frac{\mathrm{d} \tau}{\tau}\right) \frac{\mathrm{d} s}{s} \\
& \geq M_{3} \rho_{2} \int_{1}^{e} G\left(t_{0}, s\right) \varphi_{q}\left(\int_{\delta_{1}}^{e} H(s, \tau) \frac{\mathrm{d} \tau}{\tau}\right) \frac{\mathrm{d} s}{s}>\rho_{2} .
\end{aligned}
$$

This indicates that

$$
\|A u\|>\|u\|, \quad \text { for } u \in \partial B_{\rho_{2}} \cap P_{0} .
$$

On the other hand, by the second limit inequality in (H4), there exists $r_{2} \in\left(0, \rho_{2}\right)$ such that

$$
f(t, u) \leq \varphi_{p}\left(M_{2} u\right), \quad \forall u \in\left[0, r_{2}\right], t \in[1, e] .
$$

This, if $u \in \partial B_{r_{2}} \cap P_{0}$, implies that

$\|A u\|$

$$
\begin{aligned}
& \leq \int_{1}^{e} G(e, s) \varphi_{q}\left(\int_{1}^{e} H(s, \tau) \varphi_{p}\left(M_{2} u(\tau)\right) \frac{\mathrm{d} \tau}{\tau}\right) \frac{\mathrm{d} s}{s} \\
& \leq \int_{1}^{e} G(e, s) \varphi_{q}\left(\int_{1}^{e} H(s, \tau) \varphi_{p}\left(M_{2} r_{2}\right) \frac{\mathrm{d} \tau}{\tau}\right) \frac{\mathrm{d} s}{s} \\
& =M_{2} r_{2} \int_{1}^{e} G(e, s) \varphi_{q}\left(\int_{1}^{e} H(s, \tau) \frac{\mathrm{d} \tau}{\tau}\right) \frac{\mathrm{d} s}{s}<r_{2} .
\end{aligned}
$$


This gives

$$
\|A u\|<\|u\|, \quad \text { for } u \in \partial B_{r_{2}} \cap P_{0} .
$$

By the first limit inequality in (H4), there exist $R_{2}>\rho_{2}$ and $C_{4}>0$ such that

$$
f(t, u) \leq \varphi_{p}\left(M_{1} u+C_{4}\right), \quad \forall u \in \mathbb{R}^{+}, t \in[1, e] .
$$

Consequently, if $u \in \partial B_{R_{2}} \cap P_{0}$ with $R_{2}$ large enough, we obtain

$$
\begin{aligned}
& \|A u\| \leq \int_{1}^{e} G(e, s) \\
& \cdot \varphi_{q}\left(\int_{1}^{e} H(s, \tau) \varphi_{p}\left(M_{1} R_{2}+C_{4}\right) \frac{\mathrm{d} \tau}{\tau}\right) \frac{\mathrm{d} s}{s} \\
& =\left(M_{1} R_{2}+C_{4}\right) \int_{1}^{e} G(e, s) \varphi_{q}\left(\int_{1}^{e} H(s, \tau) \frac{\mathrm{d} \tau}{\tau}\right) \frac{\mathrm{d} s}{s} \\
& \leq \frac{1}{2} R_{2}+C_{5},
\end{aligned}
$$

where $C_{5}=C_{4} \int_{1}^{e} G(e, s) \varphi_{q}\left(\int_{1}^{e} H(s, \tau)(\mathrm{d} \tau / \tau)\right)(\mathrm{d} s / s)$. Hence, we have

$\|A u\|<\|u\|, \quad$ for $u \in \partial B_{R_{2}} \cap P_{0}$, if $\|u\| \longrightarrow \infty$.

In a word, from (39), (42), and (45) with $R_{2}>\rho_{2}>r_{2}$, Lemma 8 enables us to obtain that (1) has at least two positive solutions in $\left(\bar{B}_{R_{2}} \backslash B_{\rho_{2}}\right) \cap P_{0}$ and $\left(\bar{B}_{\rho_{2}} \backslash B_{r_{2}}\right) \cap P_{0}$. This completes the proof.

Example 13. Let

$f(t, u)$

$$
= \begin{cases}\rho_{1}^{p-1-\gamma_{1}} N_{3}^{p-1} u^{\gamma_{1}}, & u \in\left(\rho_{1},+\infty\right), t \in[1, e], \\ \rho_{1}^{p-1-\gamma_{2}} N_{3}^{p-1} u^{\gamma_{2}}, & u \in\left[0, \rho_{1}\right], t \in[1, e],\end{cases}
$$

where $\gamma_{1} \in(p-1,+\infty), \gamma_{2} \in(0, p-1)$, and $N_{3}, \rho_{1}$ are defined by (H3). Then

$$
\begin{aligned}
\liminf _{u \rightarrow+\infty} \frac{f(t, u)}{\varphi_{p}(u)} & =\liminf _{u \longrightarrow+\infty} \frac{\rho_{1}^{p-1-\gamma_{1}} N_{3}^{p-1} u^{\gamma_{1}}}{u^{p-1}}=+\infty \\
& \geq \varphi_{p}\left(N_{1}\right), \\
\liminf _{u \longrightarrow 0^{+}} \frac{f(t, u)}{\varphi_{p}(u)} & =\liminf _{u \longrightarrow 0^{+}} \frac{\rho_{1}^{p-1-\gamma_{2}} N_{3}^{p-1} u^{\gamma_{2}}}{u^{p-1}}=+\infty \\
& \geq \varphi_{p}\left(N_{2}\right) .
\end{aligned}
$$

Moreover, for $u \in\left[0, \rho_{1}\right], t \in[1, e]$ we have

$$
f(t, u) \leq \rho_{1}^{p-1-\gamma_{2}} N_{3}^{p-1} \rho_{1}^{\gamma_{2}}=\left(N_{3} \rho_{1}\right)^{p-1} .
$$

Therefore, (H1)-(H3) hold.

Example 14. Let

$$
f(t, u)= \begin{cases}\left(\log \delta_{1}\right)^{-(\alpha-1) \gamma_{3}} \rho_{2}^{p-1-\gamma_{3}} M_{3}^{p-1} u^{\gamma_{3}}, & u \in\left[\left(\log \delta_{1}\right)^{\alpha-1} \rho_{2},+\infty\right), t \in[1, e], \\ \left(\log \delta_{1}\right)^{-(\alpha-1) \gamma_{4}} \rho_{2}^{p-1-\gamma_{4}} M_{3}^{p-1} u^{\gamma_{4}}, & u \in\left[0,\left(\log \delta_{1}\right)^{\alpha-1} \rho_{2}\right), t \in[1, e],\end{cases}
$$

where $\gamma_{3} \in(0, p-1), \gamma_{4} \in(p-1,+\infty)$, and $M_{3}, \rho_{2}$ are defined by (H5). Then

$$
\begin{aligned}
& \limsup _{u \rightarrow+\infty} \frac{f(t, u)}{\varphi_{p}(u)} \\
& \quad=\limsup _{u \longrightarrow+\infty} \frac{\left(\log \delta_{1}\right)^{-(\alpha-1) \gamma_{3}} \rho_{2}^{p-1-\gamma_{3}} M_{3}^{p-1} u^{\gamma_{3}}}{u^{p-1}}=0 \\
& \quad \leq \varphi_{p}\left(M_{1}\right), \\
& \limsup _{u \rightarrow 0^{+}} \frac{f(t, u)}{\varphi_{p}(u)} \\
& \quad=\limsup _{u \longrightarrow 0^{+}} \frac{\left(\log \delta_{1}\right)^{-(\alpha-1) \gamma_{4}} \rho_{2}^{p-1-\gamma_{4}} M_{3}^{p-1} u^{\gamma_{4}}}{u^{p-1}}=0 \\
& \leq \varphi_{p}\left(M_{2}\right) .
\end{aligned}
$$

Moreover, for $u \in\left[\left(\log \delta_{1}\right)^{\alpha-1} \rho_{2}, \rho_{2}\right], t \in\left[\delta_{1}, e\right]$ we have

$$
f(t, u) \geq\left(\log \delta_{1}\right)^{-(\alpha-1) \gamma_{3}} \rho_{2}^{p-1-\gamma_{3}} M_{3}^{p-1} u^{\gamma_{3}}
$$

$$
=\left(M_{3} \rho_{2}\right)^{p-1}
$$

Therefore, (H1), (H4)-(H5) hold.

\section{Nontrivial Solutions for (1)}

In this section we consider the boundary value problem (1) without the $p$-Laplacian, i.e., $p=2$. In this case, (1) can be transformed into its integral form as follows:

$$
\begin{aligned}
u(t) & =\int_{1}^{e} G(t, s) \int_{1}^{e} H(s, \tau) f(\tau, u(\tau)) \frac{\mathrm{d} \tau}{\tau} \frac{\mathrm{d} s}{s} \\
& =\int_{1}^{e} G_{1}(t, s) f(s, u(s)) \frac{\mathrm{d} s}{s}, \quad \text { for } t \in[1, e] .
\end{aligned}
$$

As said in Section 3, we define an operator, still denoted by $A$, as follows:

$$
(A u)(t)=\int_{1}^{e} G_{1}(t, s) f(s, u(s)) \frac{\mathrm{d} s}{s},
$$


In what follows, we aim to find the existence of fixed points of $A$. For this, we list our assumptions on $f$ :

(H6) $f \in C([1, e] \times \mathbb{R}, \mathbb{R})$,

(H7) There exist nonnegative functions $a(t), b(t) \in \mathscr{E}$ with $b \neq \equiv$ and $K(u) \in C\left(\mathbb{R}, \mathbb{R}^{+}\right)$such that

$$
f(t, u) \geq-a(t)-b(t) K(u), \quad \forall u \in \mathbb{R}, t \in[1, e] .
$$

Moreover,

$$
\lim _{|u| \rightarrow \infty} \frac{K(u)}{|u|}=0
$$
$[1, e]$,

(H8) $\liminf \operatorname{iu|}_{\mid u \infty}(f(t, u) /|u|)>\kappa_{1}^{-1}$, uniformly in $t \in$ $[1, e]$.

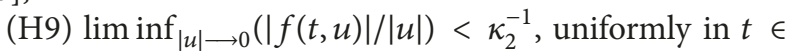

Theorem 15. Suppose that (H6)-(H9) hold. Then (1) has at least one nontrivial solution.

Proof. From (H9) there exist $\varepsilon_{3} \in\left(0, \kappa_{2}^{-1}\right)$ and $r_{3}>0$ such that

$$
|f(t, u)| \leq\left(\kappa_{2}^{-1}-\varepsilon_{3}\right)|u|, \quad \forall t \in[1, e],|u| \in\left[0, r_{3}\right)
$$

For this $r_{3}$, we show that

$$
A u \neq \mu u, \quad u \in \partial B_{r_{3}}, \mu \geq 1
$$

If otherwise, there exist $u_{1} \in \partial B_{r_{3}}, \mu_{1} \geq 1$ such that

$$
A u_{1}=\mu_{1} u_{1}
$$

and hence, we obtain

$$
\begin{aligned}
\left|u_{1}(t)\right| & =\frac{1}{\mu_{1}}\left|\left(A u_{1}\right)(t)\right| \leq\left|\left(A u_{1}\right)(t)\right| \\
& \leq \int_{1}^{e} G_{1}(t, s)\left|f\left(s, u_{1}(s)\right)\right| \frac{\mathrm{d} s}{s} \\
& \leq\left(\kappa_{2}^{-1}-\varepsilon_{3}\right) \int_{1}^{e} G_{1}(t, s)\left|u_{1}(s)\right| \frac{\mathrm{d} s}{s} .
\end{aligned}
$$

$$
R_{3} \geq \max \left\{\frac{\left(\kappa_{1}^{-1}+2\left(\varepsilon_{4}-\|b\| \epsilon\right)\right) \int_{1}^{e} W(s)\left(a(s)+\|b\| K^{*}+C_{6}\right)(\mathrm{d} s / s)}{\left(\varepsilon_{4}-\|b\| \epsilon\right) \Gamma(\alpha)-\|b\| \epsilon\left(\kappa_{1}^{-1}+2\left(\varepsilon_{4}-\|b\| \epsilon\right)\right) \int_{1}^{e} W(s)(\mathrm{d} s / s)}, \frac{\int_{1}^{e} W(s)\left(a(s)+\|b\| K^{*}+C_{6}\right)(\mathrm{d} s / s)}{\Gamma(\alpha)-\|b\| \epsilon \int_{1}^{e} W(s)(\mathrm{d} s / s)}\right\}
$$

Multiply both sides of the above inequality by $\phi(t)$ and integrate from 1 to $e$ and together with Lemma 5 we obtain

$$
\begin{aligned}
& \int_{1}^{e}\left|u_{1}(t)\right| \phi(t) \frac{\mathrm{d} t}{t} \\
& \quad \leq\left(\kappa_{2}^{-1}-\varepsilon_{3}\right) \int_{1}^{e} \int_{1}^{e} G_{1}(t, s)\left|u_{1}(s)\right| \frac{\mathrm{d} s}{s} \phi(t) \frac{\mathrm{d} t}{t} \\
& \quad \leq\left(\kappa_{2}^{-1}-\varepsilon_{3}\right) \kappa_{2} \int_{1}^{e}\left|u_{1}(t)\right| \phi(t) \frac{\mathrm{d} t}{t}
\end{aligned}
$$

This implies that $\int_{1}^{e}\left|u_{1}(t)\right| \phi(t)(\mathrm{d} t / t)=0$, and $u_{1} \equiv 0$ for the fact that $\phi(t) \not \equiv 0$, for $t \in[1, e]$, which contradicts $u_{1} \in \partial B_{r_{3}}$. Therefore, (57) is true, and from Lemma 7 we obtain

$$
\operatorname{deg}\left(I-A, B_{r_{3}}, 0\right)=1 .
$$

On the other hand, by (H8), there exist $\varepsilon_{4}>0$ and $X_{0}>0$ such that

$$
f(t, u) \geq\left(\kappa_{1}^{-1}+\varepsilon_{4}\right)|u|, \quad \forall t \in[1, e],|u|>X_{0} .
$$

For every fixed $\epsilon$ with $\|b\| \epsilon \in\left(0, \varepsilon_{4}\right),\|b\|=\max _{t \in[1, e]}|b(t)|$, and from (H7), there exists $X_{1}>X_{0}$ such that

$$
K(u) \leq \epsilon|u|, \quad \forall|u|>X_{1} .
$$

Combining the two inequalities above, $(\mathrm{H} 7)$ enables us to find

$$
\begin{aligned}
f(t, u) \geq\left(\kappa_{1}^{-1}+\varepsilon_{4}\right)|u|-a(t)-b(t) K(u) & \\
\geq & \left(\kappa_{1}^{-1}+\varepsilon_{4}\right)|u|-a(t)-\epsilon b(t)|u| \\
\geq & \left(\kappa_{1}^{-1}+\varepsilon_{4}-\|b\| \epsilon\right)|u|-a(t), \\
& \forall|u|>X_{1}, \quad t \in[1, e] .
\end{aligned}
$$

If we take $C_{6}=\left(\kappa_{1}^{-1}+\varepsilon_{4}-\|b\| \epsilon\right) X_{1}+\max _{t \in[1, e],|u| \leq X_{1}}|f(t, u)|$, $K^{*}=\max _{|u| \leq X_{1}} K(u)$. Then we easily have

$$
\begin{aligned}
f(t, u) \geq\left(\kappa_{1}^{-1}+\varepsilon_{4}-\|b\| \epsilon\right)|u|-a(t)-C_{6}, & \\
& \forall u \in \mathbb{R}, t \in[1, e] .
\end{aligned}
$$

Note that $\epsilon$ can be chosen arbitrarily small, and we let where $W(s)=\int_{1}^{e}(1-\log \tau)^{\alpha-2} H(\tau, s)(\mathrm{d} \tau / \tau)$, for $s \in[1, e]$. Now we prove that

$$
u-A u \neq \mu \phi, \quad \forall u \in \partial B_{R_{3}}, \mu \geq 0,
$$

where $\phi$ is defined by (19). Indeed, if (67) is not true, then there exists $u_{2} \in \partial B_{R_{3}}$ and $\mu_{0}>0$ such that

$$
u_{2}-A u_{2}=\mu_{0} \phi \text {. }
$$

Let $\widetilde{u}(t)=\int_{1}^{e} G_{1}(t, s)\left[a(s)+b(s) K\left(u_{2}(s)\right)+C_{6}\right](\mathrm{d} s / s)$. Then $\tilde{u} \in P_{0}$ and

$$
\begin{gathered}
\widetilde{u}(t)=\int_{1}^{e} G_{1}(t, s)\left[a(s)+b(s) K\left(u_{2}(s)\right)+C_{6}\right] \frac{\mathrm{d} s}{s} \\
\leq \int_{1}^{e} \int_{1}^{e} \frac{1}{\Gamma(\alpha)}(\log t)^{\alpha-1}(1-\log \tau)^{\alpha-2} H(\tau, s) \frac{\mathrm{d} \tau}{\tau}
\end{gathered}
$$




$$
\begin{aligned}
& \cdot\left[a(s)+b(s) K\left(u_{2}(s)\right)+C_{6}\right] \frac{\mathrm{d} s}{s} \\
& \leq \frac{1}{\Gamma(\alpha)}(\log t)^{\alpha-1} \int_{1}^{e} \int_{1}^{e}(1-\log \tau)^{\alpha-2} H(\tau, s) \frac{\mathrm{d} \tau}{\tau} \\
& \cdot\left[a(s)+b(s) K\left(u_{2}(s)\right)+C_{6}\right] \frac{\mathrm{d} s}{s} \\
& =\frac{1}{\Gamma(\alpha)}(\log t)^{\alpha-1} \int_{1}^{e} W(s) \\
& \cdot\left[a(s)+b(s) K\left(u_{2}(s)\right)+C_{6}\right] \frac{\mathrm{d} s}{s} .
\end{aligned}
$$

Consequently, we have

$$
\begin{aligned}
& \|\tilde{u}\| \leq \frac{1}{\Gamma(\alpha)} \int_{1}^{e} W(s)\left[a(s)+b(s) K\left(u_{2}(s)\right)+C_{6}\right] \frac{\mathrm{d} s}{s} \\
& \leq \frac{1}{\Gamma(\alpha)} \int_{1}^{e} W(s)\left(a(s)+C_{6}\right) \frac{\mathrm{d} s}{s} \\
& +\frac{\|b\|}{\Gamma(\alpha)}\left(\int_{\left|u_{2}\right| \leq X_{1}} W(s) K\left(u_{2}(s)\right) \frac{\mathrm{d} s}{s}\right. \\
& \left.+\int_{\left|u_{2}\right|>X_{1}} W(s) K\left(u_{2}(s)\right) \frac{\mathrm{d} s}{s}\right) \leq \frac{1}{\Gamma(\alpha)} \\
& \cdot \int_{1}^{e} W(s)\left(a(s)+\|b\| K^{*}+C_{6}\right) \frac{\mathrm{d} s}{s}+\frac{\|b\| \epsilon}{\Gamma(\alpha)} \\
& \cdot \int_{\left|u_{2}\right|>X_{1}} W(s)\left|u_{2}(s)\right| \frac{\mathrm{d} s}{s} \leq \frac{1}{\Gamma(\alpha)} \\
& \cdot \int_{1}^{e} W(s)\left(a(s)+\|b\| K^{*}+\|b\| \epsilon R_{3}+C_{6}\right) \frac{\mathrm{d} s}{s} .
\end{aligned}
$$

Plus $\tilde{u}$ into (68) gives

$$
\begin{aligned}
& u_{2}(t)+\widetilde{u}(t)=\left(A u_{2}\right)(t)+\widetilde{u}(t)+\mu_{0} \phi(t) \\
& =\int_{1}^{e} G_{1}(t, s)\left[f\left(s, u_{2}(s)\right)+a(s)+b(s) K\left(u_{2}(s)\right)\right. \\
& \left.\quad+C_{6}\right] \frac{\mathrm{d} s}{s}+\mu_{0} \phi(t) .
\end{aligned}
$$

Note that $f\left(s, u_{2}(s)\right)+a(s)+b(s) K\left(u_{2}(s)\right)+C_{6} \in P, s \in[1, e]$ and $\phi \in P_{0}$. Lemma 9 enables us to know that $u_{2}+\tilde{u} \in P_{0}$. From (65) we have

$$
\begin{aligned}
& \left(A u_{2}\right)(t)+\tilde{u}(t)=\int_{1}^{e} G_{1}(t, s)\left[f\left(s, u_{2}(s)\right)+a(s)\right. \\
& \left.+b(s) K\left(u_{2}(s)\right)+C_{6}\right] \frac{\mathrm{d} s}{s} \geq \int_{1}^{e} G_{1}(t, s) \\
& \cdot\left[f\left(s, u_{2}(s)\right)+a(s)+C_{6}\right] \frac{\mathrm{d} s}{s} \geq \int_{1}^{e} G_{1}(t, s) \\
& \cdot\left(\kappa_{1}^{-1}+\varepsilon_{4}-\|b\| \epsilon\right)\left|u_{2}(s)\right| \frac{\mathrm{d} s}{s} \geq \int_{1}^{e} G_{1}(t, s)\left(\kappa_{1}^{-1}\right. \\
& \left.+\varepsilon_{4}-\|b\| \epsilon\right) u_{2}(s) \frac{\mathrm{d} s}{s} .
\end{aligned}
$$

On the other hand, we have

$$
\begin{aligned}
\kappa_{1}^{-1} \int_{1}^{e} G_{1}(t, s)\left[u_{2}(s)+\tilde{u}(s)\right] \frac{\mathrm{d} s}{s} \\
\quad+\left(\varepsilon_{4}-\|b\| \epsilon\right) \int_{1}^{e} G_{1}(t, s) u_{2}(s) \frac{\mathrm{d} s}{s} \\
\quad-\kappa_{1}^{-1} \int_{1}^{e} G_{1}(t, s) \tilde{u}(s) \frac{\mathrm{d} s}{s} \\
\geq \kappa_{1}^{-1} \int_{1}^{e} G_{1}(t, s)\left[u_{2}(s)+\tilde{u}(s)\right] \frac{\mathrm{d} s}{s} .
\end{aligned}
$$

This inequality holds if

$$
\begin{aligned}
\left(\varepsilon_{4}-\|b\| \epsilon\right) \int_{1}^{e} G_{1}(t, s) u_{2}(s) \frac{\mathrm{d} s}{s} \\
-\kappa_{1}^{-1} \int_{1}^{e} G_{1}(t, s) \tilde{u}(s) \frac{\mathrm{d} s}{s} \geq 0 .
\end{aligned}
$$

Indeed, $u_{2}+\widetilde{u} \in P_{0}$ implies that $u_{2}(t)+\widetilde{u}(t) \geq(\log t)^{\alpha-1} \| u_{2}+$ $\tilde{u} \| \geq(\log t)^{\alpha-1}\left(\left\|u_{2}\right\|-\|\tilde{u}\|\right)$, for $t \in[1, e]$. Consequently,

$$
\begin{aligned}
& \left(\varepsilon_{4}-\|b\| \epsilon\right) \int_{1}^{e} G_{1}(t, s)\left[u_{2}(s)+\tilde{u}(s)\right] \frac{\mathrm{d} s}{s} \\
& -\left(\kappa_{1}^{-1}+\varepsilon_{4}-\|b\| \epsilon\right) \int_{1}^{e} G_{1}(t, s) \tilde{u}(s) \frac{\mathrm{d} s}{s} \\
& \geq\left(\varepsilon_{4}-\|b\| \epsilon\right)\left(R_{3}-\|\tilde{u}\|\right) \int_{1}^{e} G_{1}(t, s)(\log s)^{\alpha-1} \frac{\mathrm{d} s}{s} \\
& -\frac{\kappa_{1}^{-1}+\varepsilon_{4}-\|b\| \epsilon}{\Gamma(\alpha)} \\
& \cdot \int_{1}^{e} W(s)\left(a(s)+\|b\| K^{*}+\|b\| \epsilon R_{3}+C_{6}\right) \frac{\mathrm{d} s}{s} \\
& \cdot \int_{1}^{e} G_{1}(t, s)(\log s)^{\alpha-1} \frac{\mathrm{d} s}{s} \geq 0 .
\end{aligned}
$$

As a result, we have

$$
\begin{aligned}
\left(A u_{2}\right)(t)+\widetilde{u}(t) & \geq \kappa_{1}^{-1} \int_{1}^{e} G_{1}(t, s)\left[u_{2}(s)+\widetilde{u}(s)\right] \frac{\mathrm{d} s}{s} \\
& :=\kappa_{1}^{-1} T\left(u_{2}+\widetilde{u}\right)(t), \quad \forall t \in[1, e],
\end{aligned}
$$

where $(T u)(t)=\int_{1}^{e} G_{1}(t, s) u(s)(\mathrm{d} s / s)$, for $u \in \mathscr{E}, t \in[1, e]$. Using (68) we obtain

$$
\begin{aligned}
u_{2}+\widetilde{u} & =A u_{2}+\widetilde{u}+\mu_{0} \phi \geq \kappa_{1}^{-1} T\left(u_{2}+\widetilde{u}\right)+\mu_{0} \phi \\
& \geq \mu_{0} \phi .
\end{aligned}
$$

Define

$$
\mu^{*}=\sup \left\{\mu>0: u_{2}+\tilde{u} \geq \mu \phi\right\} .
$$

Note that $\mu_{0} \in\left\{\mu>0: u_{2}+\widetilde{u} \geq \mu \phi\right\}$, and then $\mu^{*} \geq \mu_{0}$, $u_{2}+\widetilde{u} \geq \mu^{*} \phi$. From Lemma 5 we have

$$
\kappa_{1}^{-1} T\left(u_{2}+\tilde{u}\right) \geq \mu^{*} \kappa_{1}^{-1} T \phi \geq \mu^{*} \phi,
$$


and hence

$$
u_{2}+\widetilde{u} \geq \kappa_{1}^{-1} T\left(u_{2}+\widetilde{u}\right)+\mu_{0} \phi \geq\left(\mu_{0}+\mu^{*}\right) \phi,
$$

which contradicts the definition of $\mu^{*}$. Therefore, (67) holds, and from Lemma 6 we obtain

$$
\operatorname{deg}\left(I-A, B_{R_{3}}, 0\right)=0 .
$$

This, together with (61), implies that

$$
\begin{aligned}
& \operatorname{deg}\left(I-A, B_{R_{3}} \backslash \bar{B}_{r_{3}}, 0\right) \\
& \quad=\operatorname{deg}\left(I-A, B_{R_{3}}, 0\right)-\operatorname{deg}\left(I-A, B_{r_{3}}, 0\right)=-1 .
\end{aligned}
$$

Therefore the operator $A$ has at least one fixed point in $B_{R_{3}} \backslash$ $\bar{B}_{r_{3}}$, and (1) has at least one nontrivial solution. This completes the proof.

Example 16. Let $f(t, u)=a|u|-b k(u), k(u)=\ln (|u|+1), u \in$ $\mathbb{R}, t \in[1, e]$, where $a \in\left(\kappa_{1}^{-1},+\infty\right)$ and $b \in\left(a, a+\kappa_{2}^{-1}\right)$. Then $\lim _{|u| \longrightarrow+\infty}(k(u) /|u|)=0$, and $\lim _{|u| \rightarrow+\infty}((a|u|-b \ln (|u|+$ 1)) $/|u|)=a>\kappa_{1}^{-1}, \lim _{|u| \rightarrow 0}(|a| u|-b \ln (|u|+1)| /|u|)=\mid a-$ $b \mid<\kappa_{2}^{-1}$. Therefore, (H6)-(H9) hold.

\section{Data Availability}

No data were used to support this study.

\section{Conflicts of Interest}

The authors declare that they have no competing interests.

\section{Acknowledgments}

This work is supported by Natural Science Foundation of Shandong Province (ZR2018MA011, ZR2018MA009, and ZR2015AM014).

\section{References}

[1] A. A. Kilbas, H. M. Srivastava, and J. J. Trujillo, Theory and Applications of Fractional Differential Equations, Elsevier, Amsterdam, The Netherlands, 2006.

[2] W. Yang, "Positive solutions for singular coupled integral boundary value problems of nonlinear Hadamard fractional differential equations," Journal of Nonlinear Sciences and Applications. JNSA, vol. 8, no. 2, pp. 110-129, 2015.

[3] G. Wang, K. Pei, R. P. Agarwal, L. Zhang, and B. Ahmad, "Nonlocal HADamard fractional boundary value problem with HADamard integral and discrete boundary conditions on a half-line," Journal of Computational and Applied Mathematics, vol. 343, pp. 230-239, 2018.

[4] K. Pei, G. Wang, and Y. Sun, "Successive iterations and positive extremal solutions for a Hadamard type fractional integrodifferential equations on infinite domain," Applied Mathematics and Computation, vol. 312, pp. 158-168, 2017.

[5] C. Zhai, W. Wang, and H. Li, "A uniqueness method to a new Hadamard fractional differential system with four-point boundary conditions," Journal of Inequalities and Applications, Paper No. 207, 16 pages, 2018.
[6] G. Wang and T. Wang, "On a nonlinear Hadamard type fractional differential equation with p-Laplacian operator and strip condition," Journal of Nonlinear Sciences and Applications. JNSA, vol. 9, no. 7, pp. 5073-5081, 2016.

[7] Y.-1. Li and S.-y. Lin, "Positive solution for the nonlinear Hadamard type fractional differential equation with $p$ Laplacian," Journal of Function Spaces and Applications, vol. 2013, Article ID 951643, 10 pages, 2013.

[8] H. Huang and W. Liu, "Positive solutions for a class of nonlinear Hadamard fractional differential equations with a parameter," Advances in Difference Equations, 13, article 96 pages, 2018.

[9] B. Ahmad and S. K. Ntouyas, "A fully Hadamard type integral boundary value problem of a coupled system of fractional differential equations," Fractional Calculus and Applied Analysis, vol. 17, no. 2, pp. 348-360, 2014.

[10] D. Vivek, K. Kanagarajan, and E. . Elsayed, "Nonlocal initial value problems for implicit differential equations with HilferHADamard fractional derivative," Nonlinear Analysis: Modelling and Control, vol. 23, no. 3, pp. 341-360, 2018.

[11] M. Benchohra, S. Bouriah, and J. J. Nieto, "Existence of periodic solutions for nonlinear implicit Hadamard's fractional differential equation," Revista de la Real Academia de Ciencias Exactas, Fisicas y Naturales. Serie A. Matematicas. RACSAM, vol. 112, no. 1, pp. 25-35, 2018.

[12] Y. Cui, "Uniqueness of solution for boundary value problems for fractional differential equations," Applied Mathematics Letters, vol. 51, pp. 48-54, 2016.

[13] Y. Cui, W. Ma, Q. Sun, and X. Su, "New uniqueness results for boundary value problem of fractional differential equation," Nonlinear Analysis: Modelling and Control, pp. 31-39, 2018.

[14] Y. Zou and G. He, "On the uniqueness of solutions for a class of fractional differential equations," Applied Mathematics Letters, vol. 74, pp. 68-73, 2017.

[15] L. Guo, L. Liu, and Y. Wu, "Iterative unique positive solutions for singular p-Laplacian fractional differential equation system with several parameters," Nonlinear Analysis: Modelling and Control, vol. 23, no. 2, pp. 182-203, 2018.

[16] Y. Zou and G. He, "A fixed point theorem for systems of nonlinear operator equations and applications to (p1,p2)-Laplacian system," Mediterranean Journal of Mathematics.

[17] J. Wu, X. Zhang, L. Liu, Y. Wu, and Y. Cui, "The convergence analysis and error estimation for unique solution of a pLaplacian fractional differential equation with singular decreasing nonlinearity," Boundary Value Problems, 15, article 82 pages, 2018.

[18] Y. Wang and J. Jiang, "Existence and nonexistence of positive solutions for the fractional coupled system involving generalized p-Laplacian," Advances in Difference Equations, 19, article 337 pages, 2017.

[19] X. Zhang, L. Liu, Y. Wu, and Y. Cui, "Entire blow-up solutions for a quasilinear $\mathrm{p}$-Laplacian Schrödinger equation with a nonsquare diffusion term," Applied Mathematics Letters, vol. 74, pp. 85-93, 2017.

[20] X. Hao, H. Wang, L. Liu, and Y. Cui, "Positive solutions for a system of nonlinear fractional nonlocal boundary value problems with parameters and p-Laplacian operator," Boundary Value Problems, 18, article 182 pages, 2017.

[21] J. Xu, C. S. Goodrich, and Y. Cui, "Positive solutions for a system of first-order discrete fractional boundary value problems with semipositone nonlinearities," Revista de la Real Academia de Ciencias Exactas, Físicas y Naturales. Serie A. Matemáticas. 
[22] Y. Wang, L. Liu, X. Zhang, and Y. Wu, "Positive solutions of an abstract fractional semipositone differential system model for bioprocesses of HIV infection," Applied Mathematics and Computation, vol. 258, pp. 312-324, 2015.

[23] Y. Wang, L. Liu, and Y. Wu, "Positive solutions for singular semipositone boundary value problems on infinite intervals," Applied Mathematics and Computation, vol. 227, pp. 256-273, 2014.

[24] Z. Zhao, "Positive solutions of semi-positone Hammerstein integral equations and applications," Applied Mathematics and Computation, vol. 219, no. 5, pp. 2789-2797, 2012.

[25] Z. Zhao, "Existence of positive solutions for 2 nth-order singular semipositone differential equations with Sturm-Liouville boundary conditions," Nonlinear Analysis: Theory, Methods \& Applications, vol. 72, no. 3-4, pp. 1348-1357, 2010.

[26] Y. Guo, "Positive solutions of second-order semipositone singular three-point boundary value problems," Electronic Journal of Qualitative Theory of Differential Equations, vol. 5, pp. 1-11, 2009.

[27] X. Qiu, J. Xu, D. O’Regan, and Y. Cui, “Positive solutions for a system of nonlinear semipositone boundary value problems with Riemann-Liouville fractional derivatives," Journal of Function Spaces, vol. 2018, Article ID 7351653, 10 pages, 2018.

[28] R. Pu, X. Zhang, Y. Cui, P. Li, and W. Wang, "Positive solutions for singular semipositone fractional differential equation subject to multipoint boundary conditions," Journal of Function Spaces, vol. 2017, Article ID 5892616, 7 pages, 2017.

[29] C. Chen, J. Xu, D. O’Regan, and Z. Fu, “Positive solutions for a system of semipositone fractional difference boundary value problems," Journal of Function Spaces, vol. 2018, Article ID 6835028, 11 pages, 2018.

[30] W. Cheng, J. Xu, and Y. Cui, "Positive solutions for a system of nonlinear semipositone fractional q-difference equations with q-integral boundary conditions," The Journal of Nonlinear Science and Applications, vol. 10, no. 08, pp. 4430-4440, 2017.

[31] H. Li and J. Sun, "Positive solutions of superlinear semipositone nonlinear boundary value problems," Computers \& Mathematics with Applications, vol. 61, no. 9, pp. 2806-2815, 2011.

[32] H. Li and J. Sun, "Positive solutions of sublinear Sturm-Liouville problems with changing sign nonlinearity," Computers and Mathematics with Applications, vol. 58, no. 9, pp. 1808-1815, 2009.

[33] Y. Cui, "Computation of topological degree in ordered banach spaces with lattice structure and applications," Applications of Mathematics, vol. 58, no. 6, pp. 689-702, 2013.

[34] Y. Wang, L. Liu, and Y. Wu, "Positive solutions for a class of fractional boundary value problem with changing sign nonlinearity," Nonlinear Analysis, vol. 74, no. 17, pp. 6434-6441, 2011.

[35] J. Liu and Z. Zhao, "Multiple positive solutions for secondorder three-point boundary-value problems with sign changing nonlinearities," Electronic Journal of Differential Equations, vol. 152, pp. 1-7, 2012.

[36] X. Zhang, L. Liu, and Y. Zou, "Fixed-point theorems for systems of operator equations and their applications to the fractional differential equations," Journal of Function Spaces, vol. 2018, Article ID 7469868, 9 pages, 2018.

[37] Y. Wu and Z. Zhao, "Positive solutions for third-order boundary value problems with change of signs," Applied Mathematics and Computation, vol. 218, no. 6, pp. 2744-2749, 2011.
[38] K. M. Zhang, "On a sign-changing solution for some fractional differential equations," Boundary Value Problems, 8, article 59 pages, 2017.

[39] K. Zhang, "Nontrivial solutions of fourth-order singular boundary value problems with sign-changing nonlinear terms," Topological Methods in Nonlinear Analysis, vol. 40, no. 1, pp. 5370, 2012.

[40] W. Fan, X. Hao, L. Liu, and Y. Wu, "Nontrivial solutions of singular fourth-order Sturm-Liouville boundary value problems with a sign-changing nonlinear term," Applied Mathematics and Computation, vol. 217, no. 15, pp. 6700-6708, 2011.

[41] X. Hao, M. Zuo, and L. Liu, "Multiple positive solutions for a system of impulsive integral boundary value problems with sign-changing nonlinearities," Applied Mathematics Letters, vol. 82, pp. 24-31, 2018.

[42] L. Liu, B. Liu, and Y. Wu, "Nontrivial solutions for higherorder m-point boundary value problem with a sign-changing nonlinear term," Applied Mathematics and Computation, vol. 217, no. 8, pp. 3792-3800, 2010.

[43] Y. Guo, "Nontrivial solutions for boundary-value problems of nonlinear fractional differential equations," Bulletin of the Korean Mathematical Society, vol. 47, no. 1, pp. 81-87, 2010.

[44] Y. Guo, "Nontrivial periodic solutions of nonlinear functional differential systems with feedback control," Turkish Journal of Mathematics, vol. 34, no. 1, pp. 35-44, 2010.

[45] M. Zuo, X. Hao, L. Liu, and Y. Cui, "Existence results for impulsive fractional integro-differential equation of mixed type with constant coefficient and antiperiodic boundary conditions," Boundary Value Problems, vol. 2017, article no. 161, 15, article 161 pages, 2017.

[46] X. Zhang, L. Liu, Y. Wu, and Y. Zou, "Existence and uniqueness of solutions for systems of fractional differential equations with Riemann-Stieltjes integral boundary condition," Advances in Difference Equations, 15, article 204 pages, 2018.

[47] Z. Bai and Y. Zhang, "Solvability of fractional three-point boundary value problems with nonlinear growth," Applied Mathematics and Computation, vol. 218, no. 5, pp. 1719-1725, 2011.

[48] Y. Zhang, Z. Bai, and T. Feng, "Existence results for a coupled system of nonlinear fractional three-point boundary value problems at resonance," Computers \& Mathematics with Applications, vol. 61, no. 4, pp. 1032-1047, 2011.

[49] X. Zhang, L. Liu, Y. Wu, and Y. Cui, "New result on the critical exponent for solution of an ordinary fractional differential problem," Journal of Function Spaces, vol. 2017, Article ID 3976469, 4 pages, 2017.

[50] D. J. Guo and V. Lakshmikantham, Nonlinear Problems in Abstract Cones, Academic Press, Orlando, Florida, Fla, USA, 1988. 


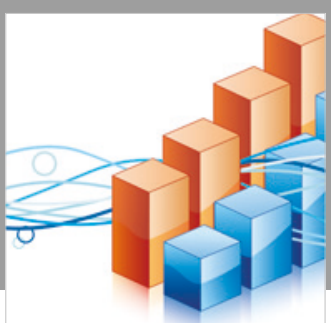

Advances in

Operations Research

\section{-n-m}
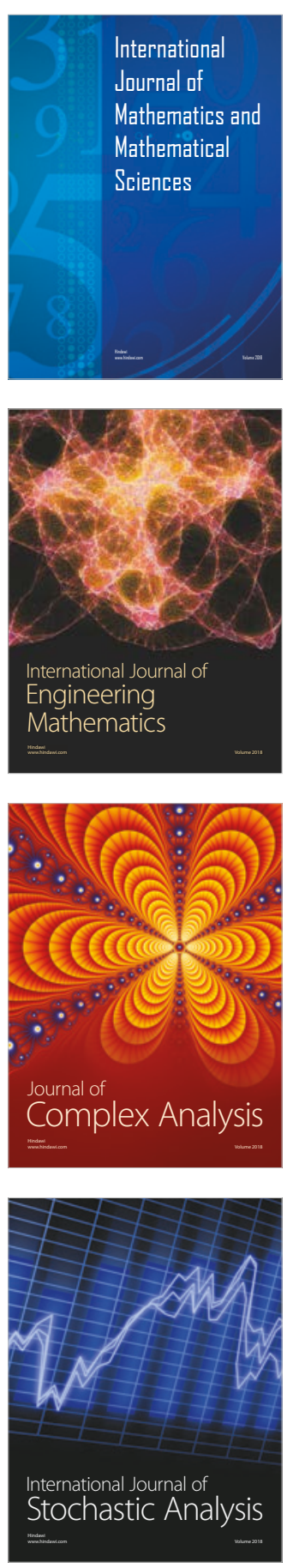
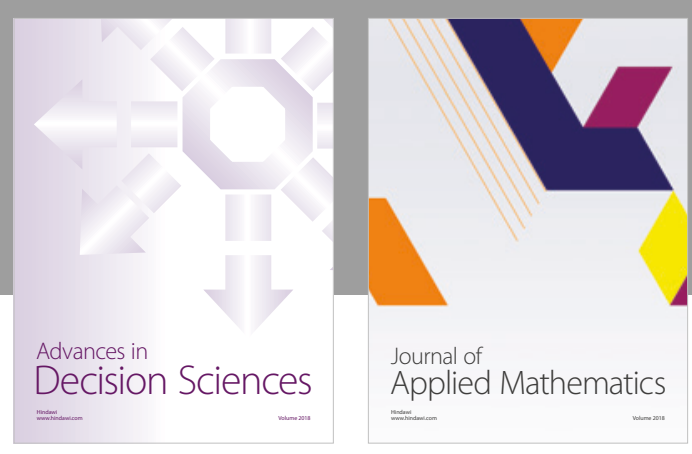

Journal of

Applied Mathematics
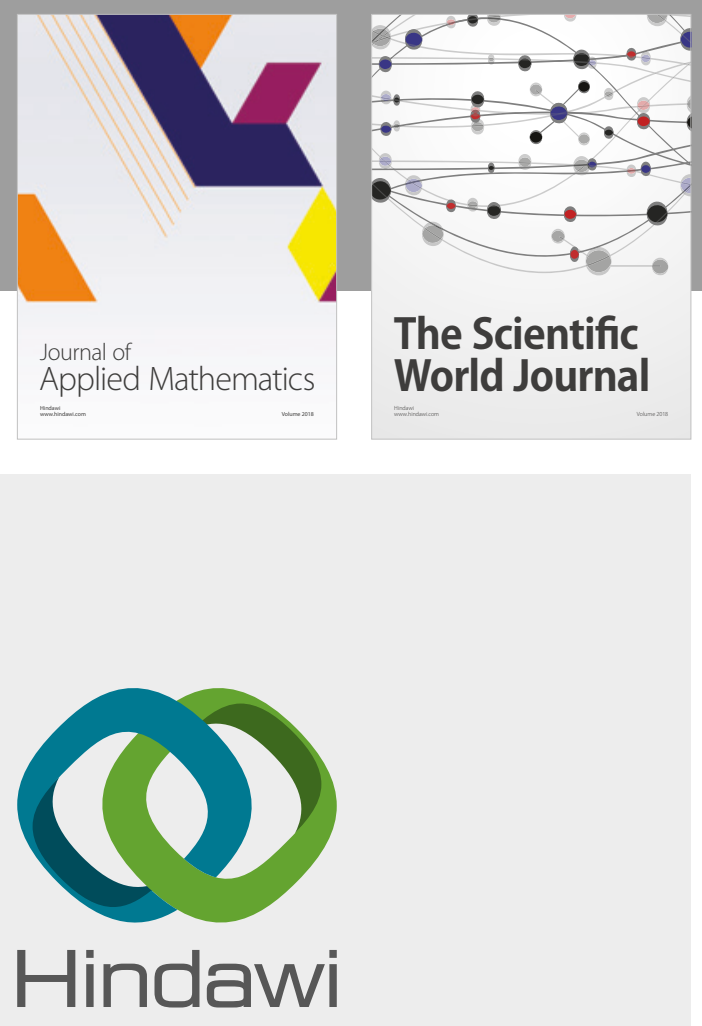

Submit your manuscripts at

www.hindawi.com

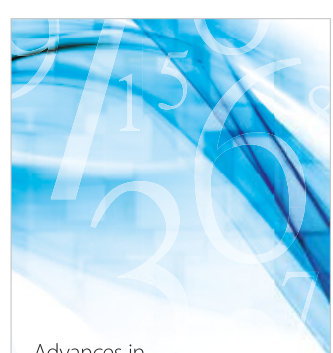

Advances in
Numerical Analysis
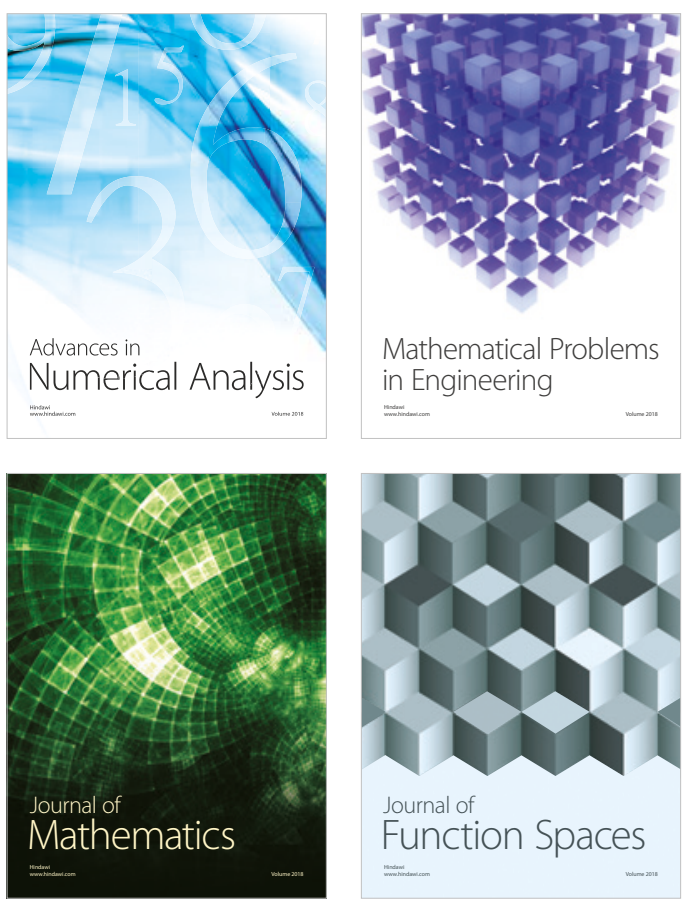

Mathematical Problems in Engineering

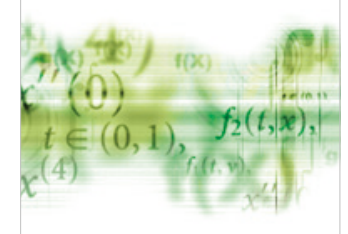

International Journal of

Differential Equations

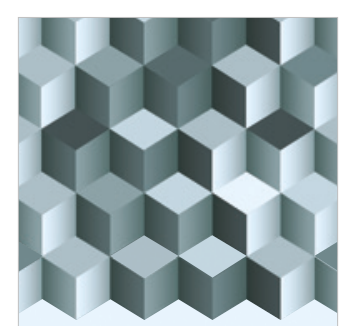

Journal of

Function Spaces

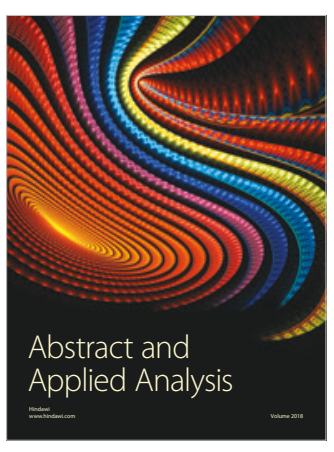

The Scientific

World Journal

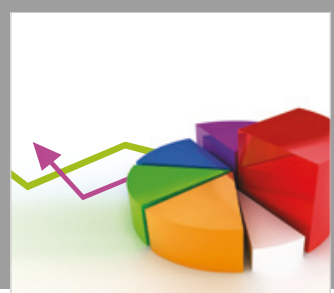

Journal of

Probability and Statistics
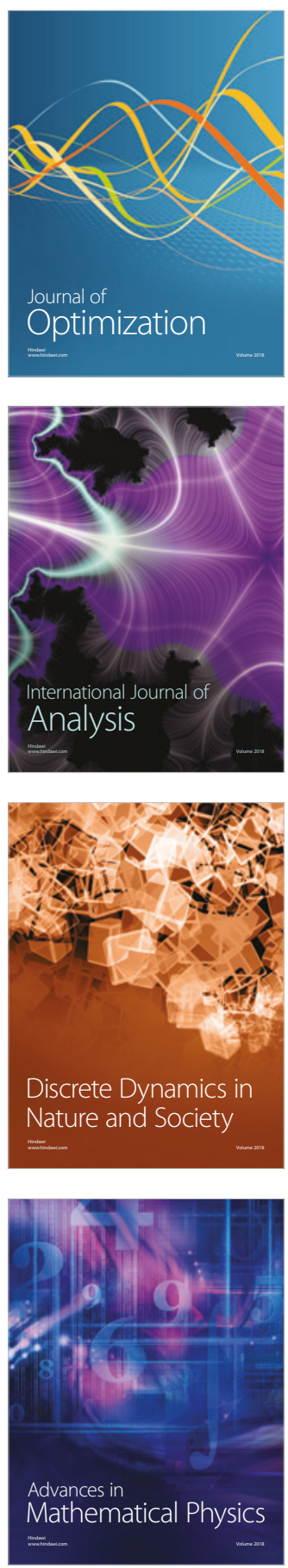